\title{
Influence of glucocorticoids on the osteogenic differentiation of rat bone marrow-derived mesenchymal stem cells
}

\author{
Da-An Zhou ${ }^{1,2}$, Hong-Xin Zheng ${ }^{3}$, Cheng-Wen Wang ${ }^{2}$, Dan Shi ${ }^{2}$ and Jian-Jun Li ${ }^{1 *}$
}

\begin{abstract}
Background: Glucocorticoid has been used extensively in clinical applications, because of its several pharmacologic actions, which include immunosuppression, anti-inflammation, anti-shock, and relief of asthma. However, the long-term or high-dose application of glucocorticoid can induce adverse effects such as osteoporosis, which is known in this case as glucocorticoid-induced osteoporosis (GIOP). It is a secondary osteoporosis that results in easy fracturing, and even disability. Therefore it became a thorny issue.

Methods: The rat model of glucocorticoid-induced osteoporosis (GIOP) was replicated to isolate BMSCs. Rats were assigned into four groups: normal, normal induction, GIOP, and GIOP induction. The growth cycle was monitored by using flow cytometry. Osteogenic differentiation was compared by using alkaline phosphatase (ALP) staining with a modified calcium cobalt method. The quantitative detection of osteoprotegerin and the receptor activator of nuclear factor kappa-B ligand (RANKL) was conducted by using enzyme-linked immunoassay. Finally, renal Klotho mRNA expression was assessed by using RT-PCR.
\end{abstract}

Results: BMSC proliferation was reduced in GIOP rats. The ALP-positive expression of normal BMSCs to the osteogenic induction solution was stronger than that of BMSCs from GIOP rats $(P<0.01)$. Osteoprotegerin expression was significantly higher in the normal induction group than in the normal, GIOP $(P<0.01)$, and GlOP induction groups $(P<0.05)$. RANKL expression was significantly higher in the normal induction group than in the other groups $(P<0.01)$ and significantly higher in the normal group than in the GIOP and GIOP induction groups $(P<0.01)$. RT-PCR analysis showed that renal Klotho mRNA expression was significantly reduced in the GIOP group compared with the normal group $(P<0.01)$.

Conclusion: BMSC proliferation, osteogenic differentiation, and reactive activity to an osteogenic inductor were reduced in GIOP rats. Klotho mRNA expression decreased during GIOP induction.

Keywords: Glucocorticoid, Osteogenic differentiation capacity, Osteoprotegerin, RANKL, Klotho gene

\section{Background}

Osteoporosis (OP) is a systemic skeletal disease characterized by low bone mass, increased bone fragility, and high morbidity and mortality. In China, OP is one of three agerelated diseases included in the National Key Research Fund. In the United States, the treatment of fractures caused by OP is estimated to cost USD 15 billion [1]. OP can increase the incidence of fractures and is one of the

\footnotetext{
* Correspondence: dahxcn@yeah.net

'Department of Spinal and Neural Function Reconstruction, School of Rehabilitation Medicine of Capital Medical University, China Rehabilitation Research Center, No. Jiaomeibei Road Fengtai District, Beijing 100068, China Full list of author information is available at the end of the article
}

main complications of glucocorticoid (GC) application [2]. This study was carried out in strict accordance with the recommendations in the Guide for the Care and Use of Laboratory Animals of the National Institutes of Health. The animal use protocol has been reviewed and approved by the Institutional Animal Care and Use Committee (IACUC) of Liaoning Medical University.

Osteoblast (OBs) and fat cells originate from bone marrow-derived mesenchymal stem cells (BMSCs) and have the same cell phenotype. When conditions are met, OBs and fat cells increase/decrease inversely [3]. GCs promote and inhibit the differentiation of BMCSs 
into lipocytes and OBs, respectively, thus leading to coupling imbalance between OBs and osteoclasts. This phenomenon causes bone loss and OP [4]. Therefore, the development of specific drugs to reduce bone turnover rate has become an urgent task. The present study aims to investigate the influence of GC on the osteogenic differentiation of rat BMSCs and determine the pathological mechanism of GCs to establish a foundation for the prevention of glucocorticoid-induced osteoporosis (GIOP) by Chinese medicine.

\section{Methods}

\section{Animals}

A total of 20 Sprague-Dawley rats (half male and half female) aged eight months and weighing $350 \pm 10$ g were provided by the Laboratory Animal Center of Liaoning Medical University. The rats were assigned equally into four groups: normal (normal BMSCs from rats), normal induction (normal BMSCs from rats + osteogenic induction solution [5,6]), GIOP (BMSCs from GIOP rats), and GIOP induction (BMSCs from GIOP rats + osteogenic induction solution) groups.

\section{Establishment of the GIOP model}

GCs could directly inhibit the osteogenic activity of osteoblasts and reduce the bone formation, leading to loss of bone mass [7]. According to reported methods [8,9], 10 of 20 rats were randomly selected to establish the GIOP models. Dexamethasone was applied into the hind limbs of rats by intramuscular injection at concentrations of $2.5 \mathrm{mg} / \mathrm{kg}$ twice a week for 9 consecutive weeks.

\section{Isolation, cultivation, and subcultivation of BMSCs}

A constant volume of the cell culture solution was maintained at $100 \mathrm{~mL}$ under sterile conditions. The solution was fully mixed and stored at $4{ }^{\circ} \mathrm{C}$. An osteogenic differentiation induction solution composed of $10^{-8} \mathrm{~mol} / \mathrm{L} \mathrm{Dex}$, $50 \mu \mathrm{mol} / \mathrm{L}$ Vitamin C, $10 \mathrm{mmol} / \mathrm{L} \beta$-GP, and $10 \%$ fetal bovine serum was used to isolate and culture the BMSCs following the whole bone marrow adherence method. Rats were dipped in $75 \%$ ethanol for $20 \mathrm{~min}$ and anesthetized with chloral hydrate. Under aseptic conditions, the bilateral lower limb femurs of rats were obtained followed by the removal of attached fatty tissues, connective tissues, and periosteum. The femurs were placed in a small aseptic beaker and then moved on a super clean bench. Thereafter, the femurs were stored on a sterile culture dish. After washing with PBS, the bilateral mummification ends in the femur were resected. A total of $5 \mathrm{ml} \mathrm{G-DMEM} \mathrm{con-}$ taining 10\% FBS was mixed with $0.5 \mathrm{ml}$ of heparin. The marrow cavities of the femurs were rinsed by the mixed solution for three to four times. The flushing fluid was fully beaten followed by cell resuspension with G-DMEM containing 10\% FBS. The cells (density was adjusted to
$1 \times 10^{6}$ cells $/ \mathrm{ml}$ ) were inoculated in a $25 \mathrm{~cm}^{2}$ culture bottle and were then cultured $\left(37^{\circ} \mathrm{C}, 5 \% \mathrm{CO}_{2}\right.$, saturated humidity). The medium was changed every three days. After the adherent cells reached $80 \%$ to $90 \%$ fusion, the culture medium was abandoned followed by washing with PBS for three times. The preheated $\left(37^{\circ} \mathrm{C}\right)$ digestion liquid containing $0.25 \%$ trypsin and $0.02 \%$ EDTA was used for digestion at room temperature followed by passage with a ratio of $1: 2$.

\section{Determination of BMSC growth cycle and osteogenic differentiation capacity}

The passage 3 growth cycle of BMSCs from normal and GIOP rats was monitored by using flow cytometry. Osteogenic differentiation was evaluated by using alkaline phosphatase (ALP) staining with a modified calcium cobalt method. Passage 3 BMSCs from normal and GIOP rats were seeded in a 24-well culture plate at a density of $5 \times 10^{4}$ cells $/ \mathrm{mL}$ with 6 parallel wells in each group. The induction groups were incubated with the osteogenic differentiation induction solution with a final volume of $2.5 \mathrm{~mL}$ in each well. The solution was replaced every 3 days for 21 consecutive days. Glass slides were harvested for ALP staining at 7, 14, and 21 days. To quantify the number of ALP-positive cells and the total number of cells and calculate the positive staining rate, 10 non-overlapping fields of view were randomly selected under a light microscope.

\section{Determination of OPG and RANKL expression}

On day 14, cell supernatants were harvested from each induction group and stored at $-20^{\circ} \mathrm{C}$. OPG and receptor activator of nuclear factor kappa-B ligand (RANKL) expressions were determined by using ELISA (ELISA USAR\&D Systems, USA).

\section{Determination of Renal Klotho mRNA expression}

RT-PCR primers and RNA PCR Kit (AMV) Ver.3.0 kit were sourced from Takara, Dalian (China). The $600 \mathrm{bp}$ DNA ladder marker was obtained from Tianze Genetic Engineering Co. Ltd. (China). Primers were designed by using Primer Premier 5.0 according to the rat Klotho (KL) and $\beta$-actin gene sequences determined in GenBank, The PCR products of $\mathrm{KL}$ and $\beta$-actin gene were 383 and $277 \mathrm{bp}$ in length,respectively. The total RNA was extracted according to the Trizol Reagent instructions (Invitrogen, USA). The first cDNA strand was synthesized according to the Takara RT-PCR kit instructions. The reaction conditions were as follows: $42^{\circ} \mathrm{C}$ for $30 \mathrm{~min} \rightarrow 99^{\circ} \mathrm{C}$ for $5 \mathrm{~min} \rightarrow 5^{\circ} \mathrm{C}$ for $5 \mathrm{~min} \rightarrow$ store at $4^{\circ} \mathrm{C}$. The PCR-amplified products were detected by electrophoresis at $9 \mathrm{~V} / \mathrm{cm}(90 \mathrm{~V})$ for 1 hour with $3 \mu \mathrm{L}$ of $100 \mathrm{bp}$ standard molecular weight DNA ladder marker as a reference. 


\section{Statistical analysis}

Experimental data were expressed as mean \pm SD and analyzed by using SPSS 11.0 software. The electrophoresis results were analyzed by using Fluor Chem V 2.0 gel imaging analysis software (Gene Genus, Syngene Inc., USA).

\section{Results}

Comparison between the BMSC growth cycles for normal and GIOP rats

Approximately $10.10 \%$ and $5.82 \%$ of BMSCs in normal and GIOP rats were arrested at $S+G_{2}+M$, respectively (Figure $1 \mathrm{~A}$ and $\mathrm{B}$ )
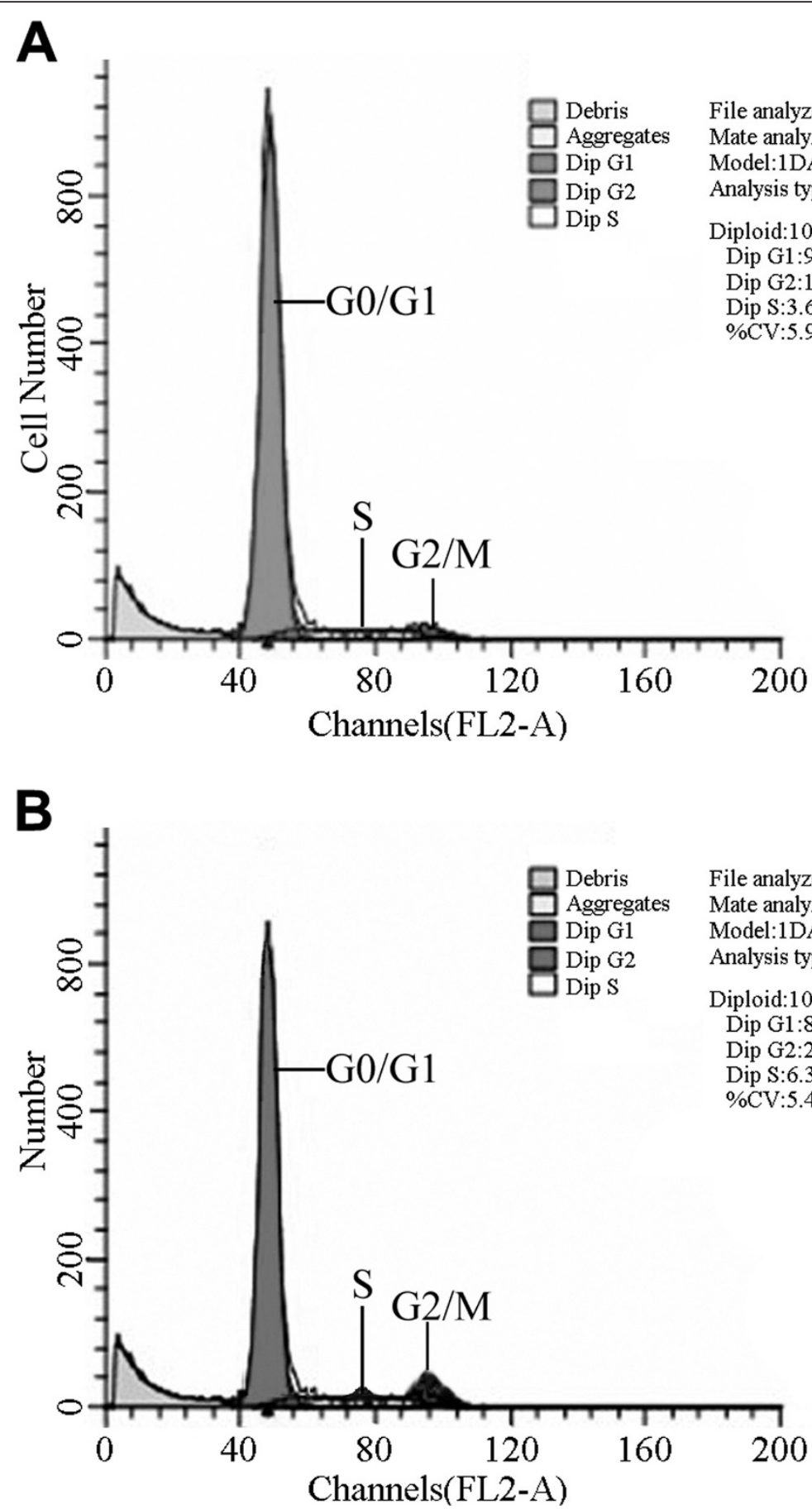

Figure 1 BMSC growth cycles for normal and GIOP rats. (A) Normal group; (B) GIOP group. 
Table 1 Comparison between the ALP-positive rates in different groups $(\bar{x} \pm \mathbf{s}, \mathbf{n}=3)$

\begin{tabular}{|c|c|c|c|}
\hline Group & $7 \mathrm{~d}$ & $14 \mathrm{~d}$ & $21 \mathrm{~d}$ \\
\hline Normal & $2.3332 \pm 0.5774^{\mathbf{\Delta ~ \Delta} \Delta \Delta}$ & $7.0011 \pm 0.9882^{\mathbf{\Delta \Delta} \Delta \Delta}$ & $8.3289 \pm 1.1632^{\mathbf{\Delta \Delta \Delta \Delta}}$ \\
\hline Normal induction & $20.3333 \pm 1.5275$ & $30.3245 \pm 1.5268$ & $30.3245 \pm 1.5268$ \\
\hline GIOP & $1.6668 \pm 0.5742^{\boldsymbol{\Delta} \Delta \Delta \Delta}$ & $2.3298 \pm 0.5699^{\bullet \bullet \Delta \Delta \Delta \Delta}$ & $3.0324 \pm 1.0349^{\bullet \bullet \Delta \Delta \Delta \Delta}$ \\
\hline GIOP induction & $12.6659 \pm 0.5543^{\boldsymbol{\Lambda}} \boldsymbol{\Lambda}$ & $21.2969 \pm 2.1375^{\boldsymbol{\Lambda}} \boldsymbol{\Lambda}$ & $31.6596 \pm 1.54688^{\boldsymbol{\Lambda}}$ \\
\hline
\end{tabular}

${ }^{\circ} P<0.05,{ }^{\bullet} P<0.01$, vs. normal group; ${ }^{\mathbf{\Delta}} P<0.05,{ }^{\mathbf{\Lambda}} P<0.01$, vs. normal induction group; ${ }^{\wedge} P<0.05,{ }^{\Delta \triangle} P<0.01$, vs. GlOP induction group.

\section{Comparison between osteogenic differentiation capacities} The ALP-positive rates at the 7 th, 14th, and 21st days in the normal induction group were significantly higher than the normal and GIOP groups, respectively $(P<0.01)$ (Table 1 and Figures 2 and 3). The ALP-positive rates at the 7 th and 14th days in the normal induction group were also significantly higher than the GIOP induction group $(P<0.01)$, except for the 21 st day of incubation $(P>0.05)$. The ALP-positive rates at the 7 th, 14th, and 21st days in the GIOP induction group were significantly higher than in the normal and GIOP groups $(P<0.01)$. The ALPpositive rates at the 14th and 21st days in the normal group were significantly higher than in the GIOP group $(P<0.01)$. These results indicated that the ALP-positive expression in normal and GIOP rats increased with the action of the osteogenic inducer; no obvious differences were observed in the responses of rats in these two groups to the osteogenic inducer at the 21st day.

\section{Comparison between OPG and RANKL expression}

OPG expression was significantly higher in the normal induction group than in the normal, GIOP $(P<0.01)$, and GIOP induction groups $(P<0.05)$. OPG expression was significantly higher in the GIOP induction group than in the GIOP $(P<0.01)$ and normal groups $(P<0.05)$. No significant difference was found between the normal and GIOP groups $(P>0.05)$.

RANKL expression was significantly higher in the normal induction group than in the other groups $(P<0.01)$. Furthermore, RANKL expression was significantly higher in the normal group than in the GIOP and GIOP induction groups $(P<0.01)$. RANKL expression was higher in the GIOP induction group than in the GIOP group but no significant differences were observed $(P>0.05$; Table 2$)$.

\section{Comparison between renal Klotho mRNA expressions in different groups}

Renal tissues from each group were amplified by using target gene and internal reference primers. Two bands with 383 and 277 bp were found as expected (Figure 4). Results from the image analysis software showed that renal KL mRNA expression was significantly reduced in the GIOP group compared with the normal group $(P<0.01)$. Table 3 shows that KL mRNA expression was significantly decreased during GIOP.

\section{Discussion}

After the transplantation of BMSCs in the GIOP rats, the growth rate of a large proportion of adipocytes and primary cells at three, four, five, and eight days was slower than the growth rate of primary cells from normal rats. Moreover, BMSCs from GIOP rats were worse than the BMSCs from normal rats with poor refraction. Approximately $10.10 \%$ of BMSCs in the normal rats were arrested at $S+G_{2}+M$ but only $5.82 \%$ of the BMSCs from the GIOP rats were arrested at $S+G_{2}+M$. Thus, the proliferation of BMSCs from GIOP rats was worse than the proliferation of normal cells.

Serum osteogenic differentiation ALP is an endocellular enzyme that can be used to evaluate the degrees of osteogenic differentiation of various cells and $\mathrm{OB}$ functions. Moreover, ALP serves as the relative specific index of the activity of OBs and the capacity of bone tissue calcification [10].

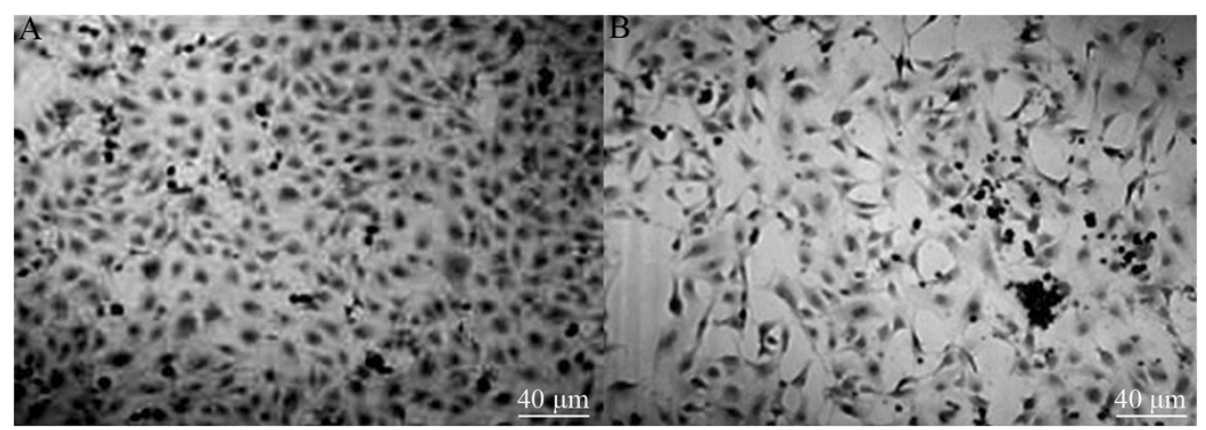

Figure 2 Osteoblasts after culturing for 14 days $(\times 100)$. (A) Normal induction group; (B) GlOP group. 


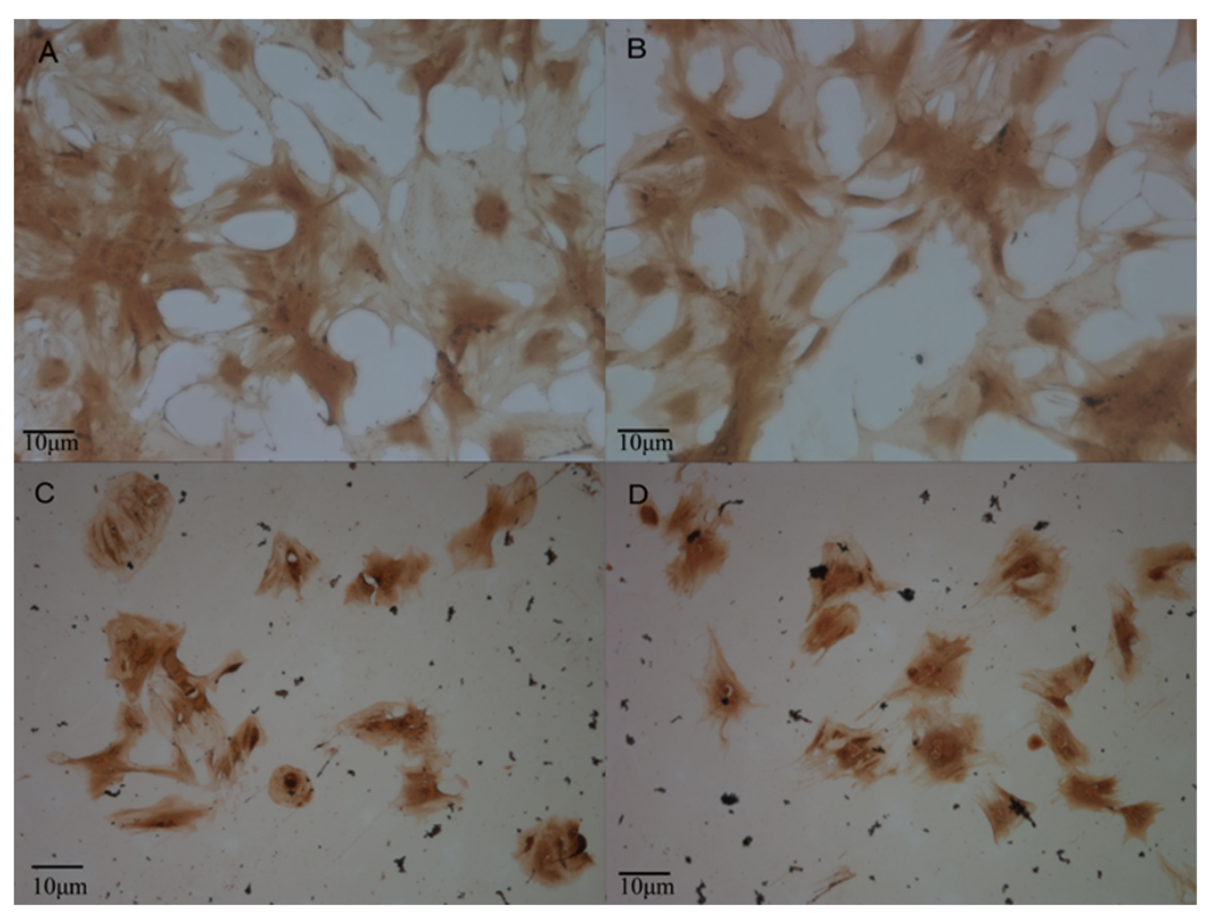

Figure 3 Comparison between the ALP-positive rates in different groups for 21 days $(\times \mathbf{4 0 0})$. (A) Normal group; (B) Normal induction group; (C) GIOP group; (D) GIOP induction group.

RANKL and OPG are recently discovered members of a superfamily of tumor necrosis factor receptors and ligands. They form the RANKL-RANK-OPG axis, which is the final pathway in the regulation of bone absorption and in vitro calcium ion balance, and plays a key role in bone formation and bone resorption coupling $[11,12]$. A variety of bone metabolism factors regulate bone formation through this axis. Studies demonstrated that OPGs isolated from humans and rats were $94 \%$ homologous and that OPG was mainly produced by OBs in bone tissues [3]. Further studies showed that OPG could inhibit osteoclast formation, differentiation, and survival and could induce $\mathrm{OB}$ apoptosis [4]. Patients with bone metabolic diseases were observed with RANK and OPG mutations [13]. GCs at physiological concentrations enhance osteoclast function by increasing the expression of parathyroid hormone receptors, whereas excessive physiological doses of GCs promote an increase in the

Table 2 Comparison between OPG and RANKL expression in different groups $(\bar{x} \pm s, n=5)$

\begin{tabular}{|c|c|c|}
\hline Group & OPG (ng/ml) & RANKL (pmol/l) \\
\hline Normal & $16.3092 \pm 0.8425^{\boldsymbol{\Delta \Lambda} \Delta}$ & $3.0708 \pm 1.2391^{\boldsymbol{\Lambda} \Lambda}$ \\
\hline Normal induction & $23.8044 \pm 1.7818$ & $5.0082 \pm 1.0355$ \\
\hline GIOP & $14.1432 \pm 2.6551 \Delta \mathbf{\Delta} \Delta \Delta$ & $1.6027 \pm 0.1123^{\bullet \bullet \Delta \Delta}$ \\
\hline GIOP induction & $20.1376 \pm 3.0025 \boldsymbol{\Lambda}$ & $2.0739 \pm 0.2375^{\bullet \bullet \Delta ~ \Delta}$ \\
\hline
\end{tabular}

${ }^{\circ} P<0.05,{ }^{\bullet \bullet} P<0.01$, vs. normal group; ${ }^{\mathbf{\Lambda}} P<0.05,{ }^{\mathbf{\Lambda}} P<0.01$, vs. normal induction group; ${ }^{\Delta} P<0.05,{ }^{\triangle} P<0.01$, vs. GIOP induction group.
RANKL/OPG ratio, thus accelerating osteoclast generation and aggravating osteoclasia [14].

In this study, we measured ALP, OPG, and RANKL expressions in BMSCs from normal and GIOP rats in the presence or absence of osteogenic induction to compare the osteogenic differentiation capacity in the different groups.

The ALP-positive expression rate results showed that the ALP-positive rates at 7, 14, and 21 days were significantly higher in the normal induction group than in the other groups $(P<0.01)$. The ALP-positive expression rates at 7,14 , and 21 days were significantly higher in the GIOP induction group than in the normal and GIOP groups $(P<0.01)$. Moreover, at 14 and 21 days, the ALPpositive rate was significantly higher in the normal group than in the GIOP group $(P<0.05)$. These results indicated that in the presence of osteogenic inductor, ALP-positive expression increased in BMSCs from both normal and GIOP rats. BMSCs from normal rats showed stronger reactive activity toward the inductor than BMSCs from GIOP rats.

OPG expression in the normal induction group was significantly higher than in the normal, GIOP $(P<0.01)$, and GIOP induction groups $(P<0.05)$. Moreover, OPG expression in the GIOP induction group was significantly higher than in the GIOP $(P<0.01)$ and normal groups $(P<0.05)$. RANKL expression was significantly higher in the normal induction group compared with 


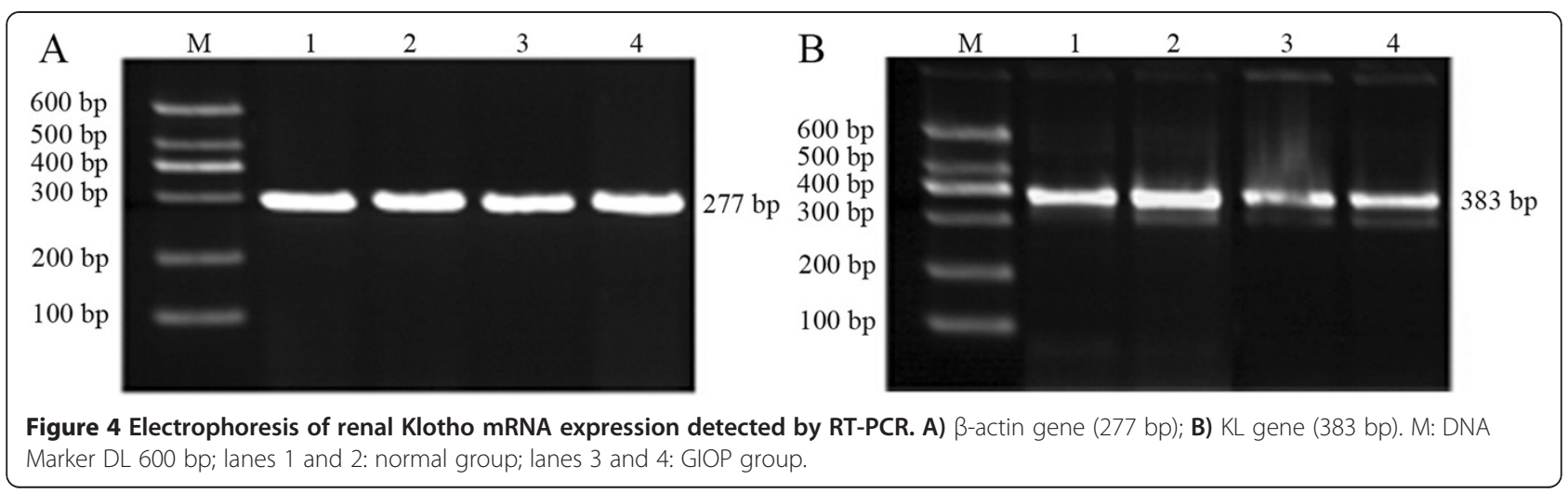

the other groups $(P<0.01)$. RANKL expression was significantly higher in the normal group compared than in the GIOP and GIOP induction groups $(P<0.01)$. These findings indicated that the osteogenic inductor could stimulate OPG and RANKL expression.

$\mathrm{KL}$ is associated with aging and was discovered by Kuro-o et al. [15]. The Kl gene in humans and mice are located in chromosome 13 (13 q12) and exhibits high homology (83\%) [16]. KL is mainly distributed in the kidneys and brain choroid. KL gene mutation or deletion can result in various phenotypes similar to human aging, such as shortened life span, arteriosclerosis, reduced immune function, and osteoporosis [17]. However, excessive $\mathrm{KL}$ expression or exogenous KL supplementation for KL-knockout mice can delay or improve aging symptoms [18]. In the experiments of the current study, renal KL mRNA expression was significantly reduced in the GIOP group compared with the normal group $(P<$ 0.01 ), thus indicating that KL mRNA expression significantly decreased during GIOP. The GC-induced reduction of KL mRNA expression may be involved in the molecular mechanism of bone metabolism.

The results of this study were consistent with previously reported results. In future studies, we will prepare drug-containing sera intervened by kidney reinforcing and marrow-benefiting Chinese medicine to investigate the effects of different blood collecting times and drugcontaining sera at different concentrations on the proliferative activity of rat BMSCs based on ALP. We will also determine the optimal BMSC proliferation-

Table 3 Influence of glucocorticoid on Klotho mRNA expression $(\bar{x} \pm s)$

\begin{tabular}{|c|c|c|c|c|}
\hline \multirow[b]{2}{*}{ Klotho } & \multicolumn{2}{|c|}{ Normal group } & \multicolumn{2}{|c|}{ GIOP group } \\
\hline & 736 & 874 & 538 & 625 \\
\hline$\beta$-actin & 1047 & 1032 & 1028 & 1040 \\
\hline Klotho/ $\beta$-actin & 0.702961 & 0.846899 & 0.523346 & 0.600962 \\
\hline $\bar{x}$ & \multicolumn{2}{|c|}{$0.7765 \pm 0.1040$} & \multicolumn{2}{|c|}{$0.5622 \pm 0.5657^{\bullet \bullet}$} \\
\hline
\end{tabular}

promoting dose and bone-directed differentiation concentration of drug-containing sera. We will use the optimal concentration to explore the possible mechanism of kidney-reinforcing Chinese medicine during the osteogenic differentiation of BMSCs.

\section{Conclusion}

BMSC proliferation, osteogenic differentiation, and reaction capacity were decreased by the osteogenic inducer. The expression of the KL gene was significantly reduced during the process of GC-induced GIOP germination.

\section{Competing interests}

The authors declare that they have no competing interests.

\section{Authors' contributions}

D-AZ participated in the experimental design, carried out isolation of MSC, culture and subculture of SD rats, participated in BMSC osteogenic differentiation testing of normal and GIOP rats, took part in statistical analysis and drafted the manuscript. $\mathrm{H}-\mathrm{XZ}$ participated in experimental design. DS and C-W Wang participated in the BMSC osteogenic differentiation testing of normal and GIOP rats. J-JL participated in the design of the study, statistical analysis and helped to draft the manuscript. All authors read and approved the final manuscript.

\section{Acknowledgements}

The comprehensive analytical study of theoretical literature and clinical information for "Kidney essence" was obtained from the Chinese national "973" plan.

\section{Author details}

${ }^{1}$ Department of Spinal and Neural Function Reconstruction, School of Rehabilitation Medicine of Capital Medical University, China Rehabilitation Research Center, No. Jiaomeibei Road Fengtai District, Beijing 100068, China. ${ }^{2}$ Department of Rehabilitation, the 3rd Affiliated Hospital of Liaoning Medical University, Jinzhou 121000, China. ${ }^{3}$ Liaoning University of Traditional Chinese Medicine, Shenyang 110032, China.

Received: 4 May 2014 Accepted: 30 June 2014

Published: 15 July 2014

\section{References}

1. Canalis E, Mazziotti G, Giustina A, Bilezikian JP: Glucocorticoid-induced osteoporosis: pathophysiology and therapy. Osteoporos Int 2007, 18:1319-1328

2. Bouxsein ML, Kaufman J, Tosi L, Cummings S, Lane J, Johnell O: Recommendations for optimal care of the fragility fracture patient to reduce the risk of future fracture. J Am Acad Orthop Surg 2004, 12:385-395. 
3. Van Staa TP, Leufkens HG, Abenhaim L, Zhang B, Cooper C: Use of oral corticosteroids and risk of fractures. J Bone Miner Res 2000, 15:993-1000.

4. Ahdjoudj S, Fromigue O, Marie PJ: Plasticity and regulation of human bone marrow stromal osteoprogenitor cells: potential implication in the treatment of age-related bone loss. Histol Histopathol 2004, 19:151-157.

5. Jin MZ, Zheng HX: Bone marrow fat cells and osteoporosis. Chin J Osteoporos 2006, 12:645-648.

6. Uemura T, Dong J, Wang Y, Kojima H, Saito T, lejima D, Kikuchi M, Tanaka J, Tateishi T: Transplantation of cultured bone cells using combinations of scaffolds and culture techniques. Biomaterials 2003, 24:2277-2286.

7. Liu F, Han H: Characteristics of rat bone marrow stroma stem cells and its differentiation to osteoblast. J Med Mol Biol 2004, 1:73-76.

8. Tian L, Liang M, Zhang J, Xian J, Huang S, Jinwei C, Luo Z: Construction of osteoporosis rats model induced by glucocorticiod. Guangxi Medical University J 2013, 30:5-6.

9. Wang J, Zheng HX, Zong ZHH, Li K, Yang F, Zhu H, Zhang G: Comparative research of the expression of osterix mrna and protein of bone tissue after using traditonal Chinese medicine to invigorate osteoporosis rats' kindney, spleen and blood induced by glucocorticoid. China J Tradit Chin Med Pharm 2011, 26:801-806.

10. Sammons J, Ahmed N, El-Sheemy M, Hassan HT: The role of BMP-6, IL-6, and BMP-4 in mesenchymal stem cell-dependent bone Development; effects on osteoblastic differentiation induced by parathyroid hormone and vitamin D(3). Stem Cells Dev 2004, 13:273-280.

11. Cao J, Venton L, Sakata T, Halloran BP: Expression of rank and OPG correlates with age-related bone loss in male C57Bl/6 mice. J Bone Res 2003, 18:270-272.

12. Kostenuik PJ, Morony S, Capparelli C, Adamu S, Shimamoto G, Shen V, Lacey DL, Dunstan CR: OPG and PTH (1-34) have additive efects on bone density and mechanical strength in osteopenic ovariectomized rats. Endocrinology 2001, 142:4295-4304.

13. Hughes AE, Ralston SH, Marken J, Bell C, MacPherson H, Wallace RG, van Hul W, Whyte MP, Nakatsuka K, Hovy L, Anderson DM: Mutations in TNFRSF11A, affecting the signal peptide of RANK, cause familial expansile osteolysis. Nat Genet 2000, 24:45-48.

14. Ikeda T, Utusuyma M, Hirokawa K: Expression profiles of receptor activator of nuclear factor $\mathrm{kB}$, and osteoprotegerin messenger RNA in aged and ovariectomized rat bones. J Bone Miner Res 2001, 16:1416-1425.

15. Kuro-o M, Matsunura $Y$, Aizawa H, Kawaguchi H, Suga T, Utsugi T, Ohyama $Y$, Kurabayashi M, Kaname T, Kume E, Iwasaki H, lida A, Shiraki-lida T, Nishikawa S, Nagai R, Nabeshima YI: Mutation of the mouse Klotho gene leads to a syndrome resembling ageing. Nature 1997, 390:45-51.

16. Xiao NM, Zhang YM, Zheng $\mathrm{Q}$, Gu J: Klotho is a serum factor related to human aging. Chin Med J (Engl) 2004, 117:742-747.

17. Nabeshima Y: Klotho: a fundamental regulator of aging. Ageing Res Rev 2002, 1:627-638.

18. Kurosu H, Yamamoto M, Clark JD, Pastor JV, Nandi A, Gurnani P, McGuinness OP, Chikuda H, Yamaguchi M, Kawaguchi H, Shimomura I, Takayama Y, Herz J, Kahn CR, Rosenblatt KP, Kuro-o M: Suppression of aging in mice by the hormone Klotho. Science 2005, 309:1829-1833.

doi:10.1186/1471-2474-15-239

Cite this article as: Zhou et al: Influence of glucocorticoids on the osteogenic differentiation of rat bone marrow-derived mesenchymal stem cells. BMC Musculoskeletal Disorders 2014 15:239.

\section{Submit your next manuscript to BioMed Central and take full advantage of:}

- Convenient online submission

- Thorough peer review

- No space constraints or color figure charges

- Immediate publication on acceptance

- Inclusion in PubMed, CAS, Scopus and Google Scholar

- Research which is freely available for redistribution

Submit your manuscript at www.biomedcentral.com/submit
Biomed Central 